\title{
Capítulo 7 Trayectorias académicas en nivel superior
}

\section{Chapter 7 Academic trajectories in higher level}

ALVAREZ-HUANTE, Claudia Guadalupe †**, MARÍN-LAREDO, Ma. Martha, LIZALDEHERNANDEZ, Azucena y DIMAS-PALACIOS, Cirila

Universidad Michoacana de San Nicolás de Hidalgo

ID $1^{\text {er }}$ Autor: Claudia Guadalupe, Alvarez-Huante / ORC ID: 0000-0002-9942-7375

ID $1^{\text {er }}$ Coautor: Ma. Martha, Marín-Laredo / ORC ID: 0000-0003-2517-3437

ID $2^{\text {do }}$ Coautor: Azucena, Lizalde-Hernandez / ORC ID: 0000-0002-7709-7141

ID $3^{\text {er }}$ Coautor: Cirila, Dimas-Palacios / ORC ID: 0000-0002-7384-4621

DOI: $10.35429 / \mathrm{H} .2019 .3 .80 .85$

C. Álvarez, M. Marín, A. Lizalde y C. Dimas

klauz_3@hotmail.com

A. Marroquín, J. Olivares, P. Diaz y L. Cruz. (Dir.) Mujeres en la innovación. Handbooks-@ECORFAN-Mexico, Querétaro, 2019. 


\begin{abstract}
General Objective: Quantify the pursuit of academic trajectories in the educational programs of the Faculty of Nursing of the Michoacán University of San Nicolás de Hidalgo. Specific Objectives: Establish a methodology to follow up on the educational programs the student is studying. Design the database to make comparisons of the 2008-2012 and 2012-2016 school years of the School of Nursing. Methodology: a quantitative, descriptive, observational, longitudinal cohort approach was used taking into account the academic indicators of the Faculty of Nursing: 1 Absorption, 2 Reprobation, 3 Dropout, 4 Retention of students, 5 Degree of titration. Contribution: Establish reference standards, in order to evaluate all aspects related to the phenomenon of school trajectory.
\end{abstract}

\title{
Students, Academic trajectory, University
}

\section{Introducción}

Hablar de trayectorias académicas como objeto de investigación es una tarea compleja, requiere claridad y reflexión dentro de los procesos educativos, es necesario realizar algunas consideraciones terminológicas de acuerdo a las trayectorias académicas, se utilizan distintos vocablos para referir a este mismo fenómeno, trayectorias académicas, trayectorias educativas y trayectorias escolares, mencionadas como sinónimos. Actualmente para evaluar un programa, incluyendo en el tema de la trayectoria, lo que más aprecian los organismos son los resultados, no el proceso que transcurre desde que ingresan y el camino que recorren para suspender, rezagarse, abandonar o egresar y titularse, es decir, la propia trayectoria (De Garay, 2017).

La base de la guía para el estudio de trayectorias académicas que aquí se plantea, es la evolución cuantitativa y formal del tránsito de los estudiantes por la universidad, esta trayectoria habrá de caracterizarse en términos de reprobación, deserción, rezago, retención de alumnos, egreso y titulación (Chain, 1997).

\section{Trayectorias Académicas}

(Rodríguez, 1997) La trayectoria escolar "se refiere a la cuantificación del comportamiento escolar de un conjunto de estudiantes (cohorte) durante su trayecto o estancia educativa o establecimiento escolar, desde el ingreso, permanencia y egreso, hasta la conclusión de los créditos y requisitos académicoadministrativos que define el plan de estudios".

De acuerdo con estas definiciones se puede afirmar que a través del conocimiento de la trayectoria escolar de los estudiantes, es posible implementar acciones para mejorar la calidad de los servicios educativos que se les ofrecen. Él termino cohorte se refiere al "conjunto de alumnos que ingresa en una carrera profesional o nivel de posgrado, en un año determinado, y que cumple un trayecto escolar en el periodo normal en que prescribe el plan de estudios". La cohorte es la unidad fundamental del análisis estadístico, porque con base en ella se pueden agrupar y desagregar los datos referentes a los alumnos (Huerta, 2015).

Aludiendo a lo anterior y de acuerdo con Altamira (Rodríguez, 1997). Refiere que las trayectorias académicas es la cuantificación del comportamiento escolar de un conjunto de estudiantes, a partir de una cohorte, durante su estancia educativa que conforma desde su ingreso hasta el egreso.

En este sentido la Facultad de Enfermería de la Universidad Michoacana de San Nicolás de Hidalgo. Realiza una reflexión acerca de los indicadores más sustanciales de las trayectorias académicas: Los indicadores que se describen a continuación son los siguientes:

$\begin{array}{ll}\text { - } & \text { Absorción } \\ \text { - } & \text { Reprobación } \\ \text { - } & \text { Deserción } \\ \text { - } & \text { Retención de alumnos } \\ \text { - } & \text { Titulación }\end{array}$


La combinación de los indicadores denominados desempeño escolar (DE), que incluye a los indicadores de Índice de Aprobación en Ordinario, Índice de Promoción y Promedio, y situación escolar (SE), nos da la posibilidad de observar de manera sintética con un solo indicador los diversos tipos de caminos o trayectorias escolares que siguen los alumnos.

De los indicadores mencionados, los tres primeros son datos básicos que sirven para construir el índice de desempeño escolar (DE); el tipo de estudiante o trayectoria escolar (TE) es la combinatoria del desempeño escolar (DE) y de la situación escolar (SE); finalmente, el riesgo de abandono es una agrupación de la trayectoria escolar, como se puede apreciar, los indicadores van de datos básicos a indicadores complejos (Ortega, López, Alarcón, 2015).

Características de planes y programas de estudio.

Los diversos diseños y modos de operación de los planes y programas de estudio pueden ofrecer características poco coincidentes en las carreras que se ofrecen en la misma DES de Ciencias de la Salud, con propósitos también diferentes que dificultan la posibilidad de un trabajo integral, promoviendo los estancos de la universidad tradicional en la que la interdisciplinariedad está ausente.

En este modelo educativo se pretenden promover alternativas que contribuyan a:

- Contar con características homogéneas que permitan la formación integral de los estudiantes.

- Tener una estructura homogénea que permita la movilidad de los estudiantes y promueva una formación interdisciplinaria.

Que los recursos humanos, materiales y técnicos con los que se cuenta en las Unidades Académicas de esta DES. sean utilizados con mayor efectividad para lograr los propósitos que se persiguen en este Modelo Educativo (UMSNH DES, 2007).

Objetivo General: Cuantificar el seguimiento de trayectorias Académicas en los programas educativos de la Facultad de Enfermería de la Universidad Michoacana de San Nicolás de Hidalgo

\section{Metodología a desarrollar}

En esta investigación se utilizó un enfoque cuantitativo, estudio descriptivo observacional de cohorte transversal utilizando dos generaciones del plan de estudios (2008-2012), así mismo establecer estándares de referencia, con el fin de evaluar todos los aspectos relativos al fenómeno de trayectoria escolar. Donde se abordan los Indicadores Académico de la Facultad de Enfermería.

Los indicadores que se describen a continuación son los siguientes:

- $\quad$ Absorción: Refiere al porcentaje de estudiantes aceptados en la Facultad de Enfermería de la Universidad Michoacana en relación al total de aspirantes al programa educativo

Reprobación: La tasa de reprobación por semestre será la proporción de alumnos que reprueban dos o más asignaturas y la matrícula del semestre escolar.

Deserción: La deserción se define como el abandono que hace el alumno de los cursos o carreras a las que se ha inscrito, dejando de asistir a las clases y de cumplir con las obligaciones establecidas previamente, lo cual afecta la eficiencia terminal del conjunto.

- $\quad$ Retención de alumnos: La tasa de retención, de acuerdo con Ramsden (1999) es el porcentaje de estudiantes (o que están terminando sus estudios) retenidos por la institución.

Tasa de titulación: Es el porcentaje de estudiantes titulados en relación a los egresados de una cohorte de ingreso, en el momento de la consulta. 


\section{Estrategias a Desarrollar}

- Diseñar la base de datos para realizar el comparativo del ciclo escolar 2008-2012 y 2012-2016 de la facultad de enfermería.

- Realizar el estudio de las trayectorias académicas desde los diseños transversales y longitudinales.

- Establecer estándares de referencia, con el fin de evaluar todos los aspectos relativos al fenómeno de trayectoria escolar.

\section{Resultados}

En este panorama, la digitalización de documentos y la automatización de los procesos de selección e ingreso son cada vez más indispensables, no sólo para optimizar los recursos institucionales sino también para un mejor manejo de la información.

Se aporta la información del total de estudiantes de nuevo ingreso con posibilidades de atender la demanda por parte de la institución, el total de la matrícula de estudiantes aceptados en los ciclos mencionados los ciclos que tuvieron más incremento se presentaron en el 2011/2012 y en segundo lugar 2010/2011. En los demás ciclos escolares la matricula se equilibró con número de estudiantes aceptados entre los 400 y 600 estudiantes. Cabe mencionar que la Facultad de Enfermería ha mantenido la absorción de su matrícula sin rebasar una masificación.

De acuerdo al indicador de reprobación en el ciclo 08/09 el porcentaje que representa es del $11.0 \%$ de estudiantes reprobados y con un $89.0 \%$ a estudiantes aprobados, asi mismo se considera una oportunidad para establecer estrategias para evitar la reprobación incluyendo un diagnostico al estudiante y docente que imparten la unidad de Aprendizaje.

Respecto al indicador de retención en la Facultad de Enfermería, en los ciclos que más masificación obtuvieron oscilan entre $08 / 11$ posteriormente se mantiene entre el indicador ideal que es 28 estudiantes por sección correspondiente a 15 secciones lo que equivale a 420 estudiantes, aunando a los estudiantes que se incorporan por reprobación o deserción en ciclos anteriores.

\section{Anexos}

Tabla 7.1 Tasa de Titulación

\begin{tabular}{|c|}
\hline$\underline{\text { ETE }=\text { ATC X 100 }}$ \\
\hline $\begin{array}{c}\text { ATC }=\text { Número de estudiantes de la cohorte } \\
\text { que se han titulado. }\end{array}$ \\
\hline $\begin{array}{l}\text { AE }=\text { Número de estudiantes de la cohorte que } \\
\text { han egresado atendiendo a los criterios ya establecidos } \\
\text { anteriormente para determinar la eficiencia de egreso y } \\
\text { la eficiencia terminal de titulación. }\end{array}$ \\
\hline
\end{tabular}

Fuente: Dirección de Planeación General UAH (2003)

Tabla 7.2 Retención de Alumnos Matriculados

\begin{tabular}{|l|l|l|l|}
\hline \multicolumn{2}{|c|}{ Ciclo } & \multicolumn{2}{c|}{ Retención de Alumnos Matriculados } \\
\hline $8 / 9$ & 143 & 624 & Total \\
\hline $9 / 10$ & 143 & 641 & 624 \\
\hline $10 / 11$ & 143 & 864 & 641 \\
\hline $11 / 12$ & 143 & 1159 & 864 \\
\hline $12 / 13$ & 143 & 519 & 1159 \\
\hline $13 / 14$ & 143 & 528 & 519 \\
\hline $14 / 15$ & 143 & 620 & 528 \\
\hline $15 / 16$ & 143 & 627 & 620 \\
\hline $16 / 17$ & 143 & 489 & 627 \\
\hline
\end{tabular}


Grafica 7.1 Total de Absorción

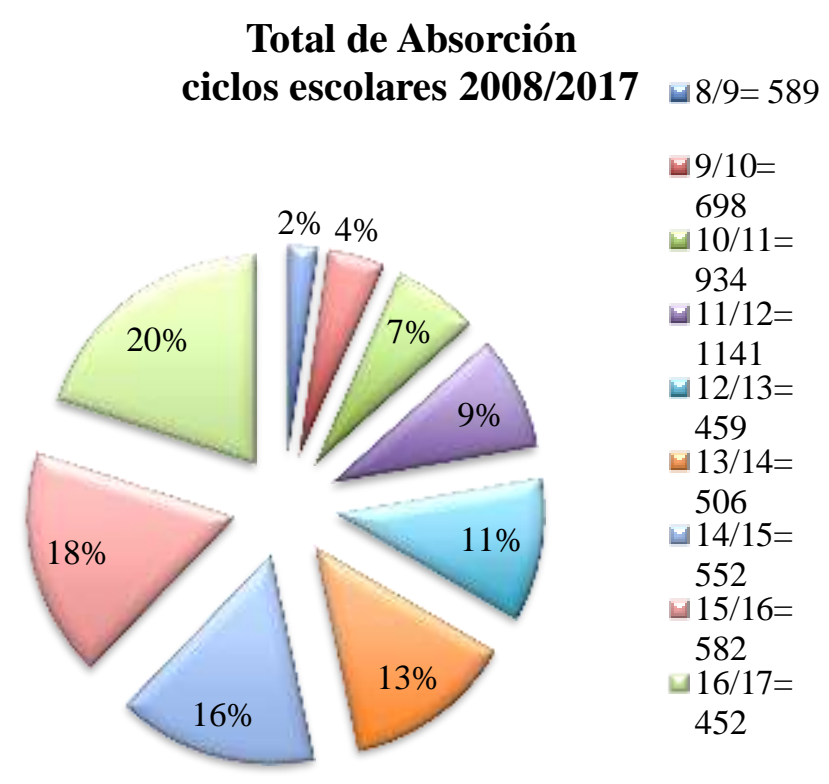

Fuente: Indicador de titulación 2012. Facultad de Enfermería

Gráfica 7.2 Indicador de Titulación

Indicador de Titulación Ciclo 2012

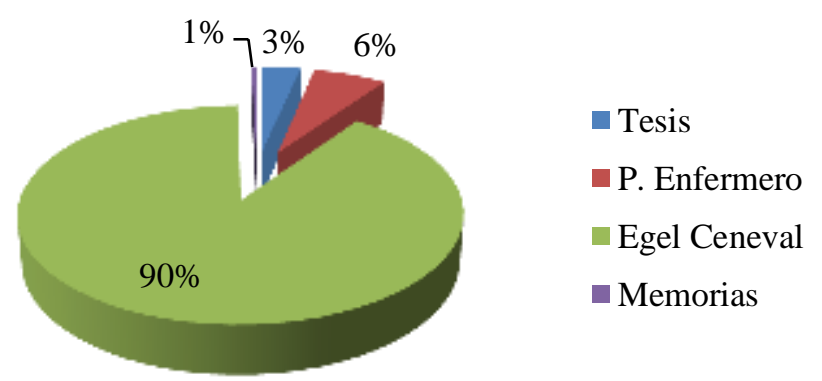

Fuente: Facultad de Enfermería

\section{Agradecimiento}

Se agradece a la Facultad de enfermería por permitir realizar este estudio cuantitativo como diagnóstico para establecer estándares de referencia, con el fin de evaluar todos los aspectos relativos al fenómeno de trayectoria escolar.

\section{Conclusiones}

El estudio de las trayectorias académicas de los estudiantes constituye una línea de investigación capaz de aportar información relevante al renovado debate sobre los fenómenos de rezago y abandono y sustentar el diseño de estrategias orientadas al logro de una educación superior más inclusiva. Atendiendo a la complejidad de este objeto de estudio, se analizan distintas perspectivas conceptuales en torno al concepto de trayectorias académicas y a su abordaje en este proceso permanente y continuo.

Finalmente al realizar esta investigaciones sobre las trayectorias escolares obtenemos información útil en distintos niveles y ámbitos, relacionados con el sector estudiantil, las trayectorias escolares proveen datos para la evaluación de los aprendizajes y de las estrategias institucionales en el proceso de enseñanza, para estudios sobre el comportamiento académico, para el análisis del desempeño escolar por género, por origen socioeconómico, o según sus antecedentes escolares, de acuerdo al objetivo planteado de estas trayectorias académicas se cumplió, sin embargo queda mucho camino por recorrer junto con las estrategias planteadas. 


\section{Referencias}

Altamira Rodríguez, A. (1997). El análisis de las trayectorias escolares como herramienta de evaluación de la actividad académica universitaria: Un modelo ad hoc para la Universidad Autónoma de Chiapas, el caso de la Escuela de Ingeniería Civil, Tesis de Maestría en Educación. Universidad Autónoma de Chiapas. México.

Chain R. y Ramírez M. (1997). Trayectoria Escolar: La Eficiencia Terminal en la Universidad Veracruzana en Revista de la Educación Superior, Núm. 102, abril- junio. ANUIES. México.

Dependencia de Educacion Superior Ciencias de la Salud. (2007). Universidad Michoacana de San Nicolas de Hidalgo.

De Garay, A. (2017). Estudiantes diversos, diferentes y desiguales. Estrategias de investigación y políticas institucionales. Congreso Nacional de Investigación Educativa. XIV. San Luis Potosí.

Huerta, I.J. (2015), en Seguimiento de Trayectorias Escolares. ANUIES. México.

Ortega, J. López, O. Alarcón, R. (2015). Trayectorias escolares en educación superior. Propuestas metodológicas y experiencias en México. Universidad Veracruzana. Biblioteca digital de investigación educativa. Xalapa, Veracruz.

Universidad michoacana de san Nicolás de Hidalgo Facultad de Enfermería. Mapa curricular plan de estudios (2008). 